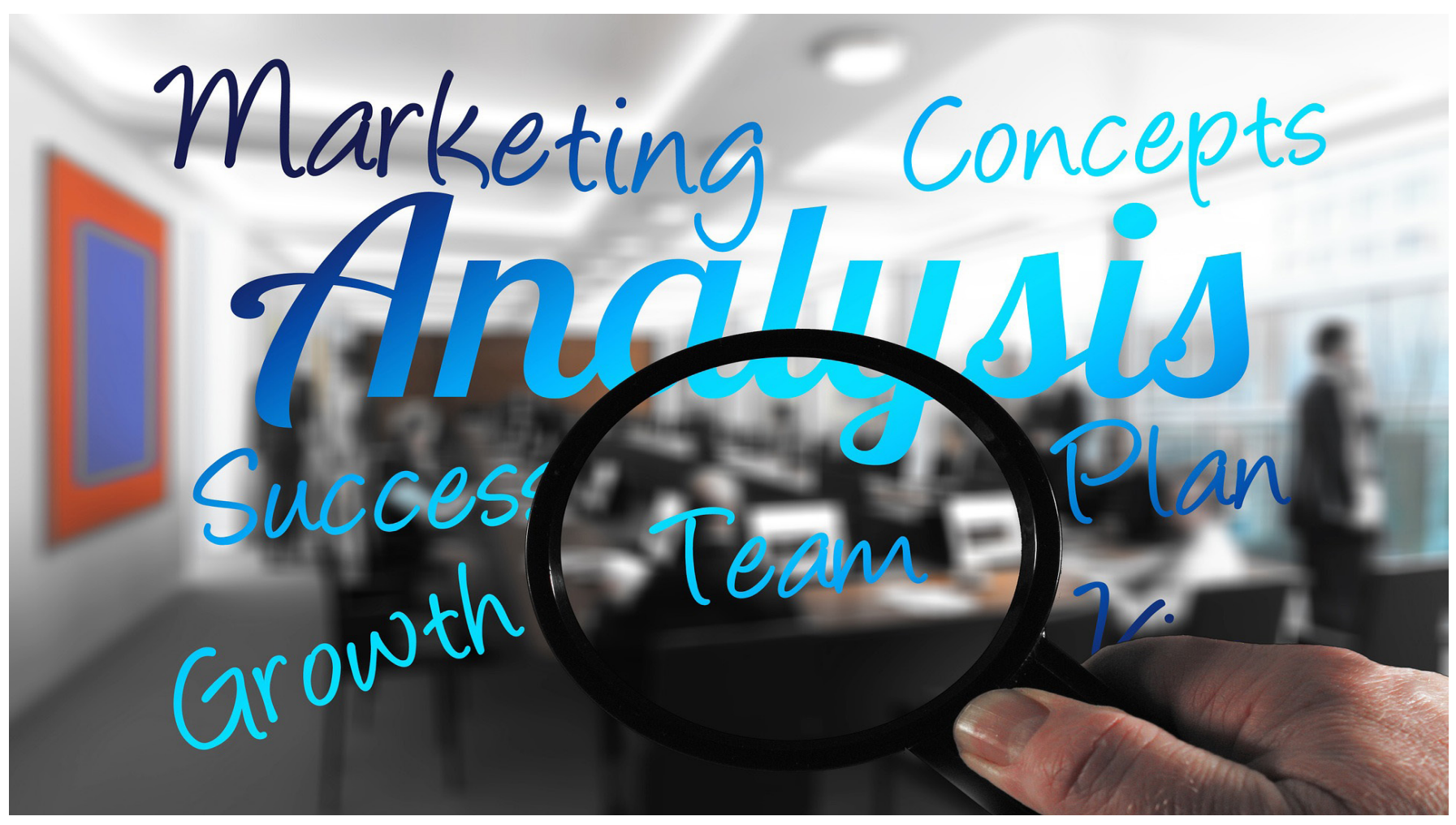

\title{
La Auditoría de Gestión y su incidencia en la rentabilidad de las Pymes
}

\section{The Management Audit and its Impact on the Profitability of SMEsPractice}

Yuliana Isabel Rivera-Espinel

Estudiantes del Programa de Contaduría Pública, Universidad Francisco de Paula Santander, Cúcuta, Colombia

Dilani Yhirley Rodríguez-Montes

Estudiantes del Programa de Contaduría Pública, Universidad Francisco de Paula Santander, Cúcuta, Colombia
Luisa Fernanda Peñaranda-Sarabia

Estudiantes del Programa de Contaduría Pública, Universidad Francisco de Paula Santander, Cúcuta, Colombia

\section{Carlos Fabian Rico-Rojas}

Magister en Dirección Estratégica Especialidad Gerencia Módulo Optativo Auditoría, carlosfabianrr@ufps.edu.co, Universidad Francisco de Paula Santander, Cúcuta, Colombia 
Cómo citar: Y.I. Rivera-Espinel, D.Y. Rodríguez-Montes, L.F. Peñaranda-Sarabia, C.F. Rico-Rojas. (2018). La Auditoría de Gestión y su incidencia en

la rentabilidad de las Pymes". Reflexiones contables (Cúcuta), 1(1), 27-35

\section{Resumen}

El presente estudio tiene el objetivo de analizar la rentabilidad e influencia que ejerce sobre las pequeñas y medianas empresas la implementación, estudio y aplicación del modelo de auditoría de gestión, con el fin de conocer el proceso de implementación y su influencia sobre las pymes se realizó documentación de literatura que tuviera por objeto explicar los criterios expuestos en un modelo de auditoría, se encontró que la auditoría es un examen crítico dentro de una empresa para determinar cómo se manejan los recursos, los resultados de procesos realizados, la auditoría como un examen representa una fuente verás de información dentro de la empresa, su enfoque de auditoría de gestión constituye un modelo capaz de evaluar y analizar información externa e interna tanto administrativa como operativa de las empresas con el fin de determinar el impacto o cómo se están utilizando y gestionando los recursos, otra de sus funciones dentro de la empresa es el análisis dentro de la toma de decisiones, ya que como es un examen crítico, se encarga de brindar resultados que hagan prever las posibles mejores decisiones para que la empresa mejore tanto sus procesos administrativos, gestión de recursos como operativos. Mediante la documentación realizada se puede concluir que la auditoría de gestión como concepto relativamente nuevo, puede brindar dentro del proceso interno de una pyme, mejorías entorno a la gestión, ya que al tener un análisis detallado permite conocer los puntos críticos que están afectando el manejo de la pyme y por lo tanto su rentabilidad, también se determinó que para que un modelo de auditoría de gestión funciones correctamente debe estar apoyado en un personal de administración ampliamente capacitado que logre la solución de los problemas detectados por el modelo, y así gestione los recursos y los procesos para el mejoramiento de la rentabilidad, aplicar el modelo de auditoría de gestión en una pyme ser parte vital dentro del crecimiento y rentabilidad de esta.

Palabras clave: Pyme, gestión, administración, estrategia, análisis, auditoría.

\section{Abstract}

The present study has the objective of analyze the profitability and influence that the implementation, study and application of the performance audit model exerts on small and medium-sized companies, in order to know the implementation process and its influence on SMEs. Literature documentation that was intended to explain the criteria exposed in an audit model, it was found that the audit is a critical examination within a company to determine how resources are managed, the results of processes performed, the audit as an examination represents a source of information within the company, its management audit approach constitutes a model capable of evaluating and analyzing external and internal information, both administrative and operational, of the companies in order to determine the impact or how they are using and managing the resources, another of its functions within the company is the analysis within decision making sions, since as it is a critical examination, it is responsible for providing results that make foresee the best possible decisions for the company to improve both its administrative, resource management and operational processes. Through the documentation carried out, it can be concluded that the management audit as a relatively new concept, can provide within the internal process of an $\mathrm{SME}$, improvements around the management, since having a detailed analysis allows knowing the critical points that are affecting the management of the SME and therefore its profitability, it was also determined that for a performance audit model to function correctly it must be supported by extensively trained administration staff who can solve the problems detected by the model, and thus manage the resources and processes for improving profitability, applying the performance audit model in an SME is a vital part of its growth and profitability.

Keywords: SME, management, administration, strategy, analysis, audit. 


\section{Introducción}

La auditoría de gestión constituye una técnica nueva de asesoramiento para el diagnóstico, análisis y recomendaciones a una empresa, con el objetivo de tener éxito en una estrategia. La auditoría de gestión analiza las operaciones administrativas de una organización con el fin de determinar la eficiencia en los procesos realizados por esta, determinando así si los objetivos previamente propuestos son alcanzados. La auditoría encargada principalmente de la información, dentro del ámbito de la gestión usa la información para obtener un diagnóstico e identificar que componentes o acciones resultan sumamente importantes dentro de las empresas, teniendo en cuenta las actitudes tácticas, las funciones desarrolladas, sus conocimientos. La auditoría de inspección en las bases del control interno establece un gran alcance, en el mejoramiento de este. Se destaca la importancia de incorporar la práctica de la auditoría como como un estándar para la gestión estratégica de las funciones y servicios, de la mano de la información en cualquier tipo de organización (Soy, 2003).

Dentro del control de gestión son principalmente evaluados los resultados, en estos resultados se busca la concordancia entre aquello que la empresa prevé ser en un futuro junto con las metas a corto plazo establecidas. La auditoría de gestión define sobre todo características, bases o normas con el fin de establecer criterios que permitan analizar el proceso de gestión y los resultados producidos dentro de las empresas. La auditoría de gestión hace parte de la estructura en una empresa, su fin propone un mejoramiento de la técnica base para enfrentar retos, cambios, evaluando las fortalezas debilidades, identificando los objetivos estratégicos. Según afirma Gras \& García (2015) "La auditoría interna es generalmente el principal responsable de controlar día a día las acciones de la dirección, así como las relacionadas con la información financiera". La auditoría en las empresas sirve para garantizar que las autoridades de gestión adopten un planteamiento eficaz, estructurado y específico para gestionar la auditoría y su información en las empresas. (Fababa, 2020). Considerando la auditoría de gestión como un modelo de solución inmediata que promueve la eficiencia, eficacia en la realización de las operaciones proporcionando de igual manera seguridad, razonamiento, determinación de costes y simplificación de tareas, con el fin de cumplir metas y objetivos formulados. Martínez (2011) y Leal (2015) señalan que "La auditoría funciona como una herramienta que evalúa la eficacia de usos de recursos, conjuntamente a la ejecución de procesos analizando la pertinencia de las estrategias aplicadas, la auditoría de gestión permite a las organizaciones el mejoramiento de procesos así como enfrentar cambios en los mercados y elevar niveles de productividad". El presente estudio se centra en determinar cómo se puede establecer la técnica de la auditoría de gestión, partiendo de la auditoría de la información, como una fuente principal de esta para una gestión correcta de la administración así como el mejoramiento del desarrollo de estrategias. La auditoría proporciona información que siendo aprovechada desde la administración y gestión, mejora tanto los procesos internos como los externos. En este sentido, formulando cómo puede esta técnica apoyar en la rentabilidad a las empresas.

\section{Desarrollo}

En la gestión administrativa de una empresa, se presentan problemáticas como una baja o negativa técnica de trabajo, esto se debe a que las empresas no gestionan correctamente cómo llevar a cabo sus procesos, mediante la auditoría interna los problemas de gestión podrían verse solucionados ya que la auditoría recopila información de extrema importancia que es analizada, de esta manera llegando a 


\section{0}

las posibles soluciones para el mejoramiento de los procesos empresariales.

La auditoría de gestión principalmente funciona como un procedimiento de asesoría, uno de sus propósitos es evaluar la función de la gestión interna de una empresa. En una Pyme existen múltiples problemas de gestión que puede incurrir en una rentabilidad en disminución, por eso es importante el aplicar técnicas que mejoren la problemática a la que está expuesta, una de estas suele ser la administración, en varias ocasiones no se cuenta con una administración especializada, y casi siempre se piden asesorías externas para la solución de problemas que deberían ser afrontados por el personal encargado de la administración, al no contar con personal capacitado es muy probable que se llegue a situaciones decrecientes para la empresa, una auditoría de gestión sería la parte calificada y especializada dentro de la empresa, es importante contar con ella pues constituye un fuerte apoyo para el departamento de administración (Ríos, 2011).

De la misma forma en que la auditoría puede significar una columna de apoyo dentro de la administración, también puede ésta ser importante en la gestión de recursos, ya que mediante un examen de auditoría de gestión se puede determinar con la medida de recursos destinados para hacer frente tanto para las empresas que desean producir y vender sus productos, como para hacer frente a los cambios en el mercado. Se destaca la auditoría de gestión por la utilización de indicadores que determinan la rentabilidad existente en una empresa. La auditoría interna es un examen encargado del análisis crítico, es realizado por un profesional especializado contratado internamente por la empresa, sus métodos técnicos son usados para la realización de informes y de esta manera la formulación de soluciones o procedimientos con el fin de mejorar la situación interna, los informes mencionados son de carácter y circulación interno (Ríos, 2011).

Por otra parte, la falta de planeación en los procesos de las Pymes el poco análisis del entorno implica que estas empresas generalmente no realizan análisis críticos del entorno, desarrollar estrategias conociendo las deficiencias en los procesos es crucial para mejorarlos y no presentar problemas en el entorno empresarial. De esta misma manera las pymes que no realizan estudios para la identificación de los problemas principales presentan enormes deficiencias, una buena planeación implicaría el uso de la auditoría como parte fundamental para gestionar interna y externamente la influencia de dichos problemas dentro del sector empresarial y la manera de disminuir los impactos, así como presentar soluciones y métodos del mejoramiento de procesos (Palomo, 2005).

Por tanto, uno de los principales problemas dentro de las pymes, son los estudios de mercado, este tema anteriormente tratado, puede deberse a la deficiencia en la planeación de la empresa. La auditoría de gestión, siendo un examen de análisis crítico, ayuda a determinar la problemática ocasionada por el mal manejo y mala planeación, así como las posibles soluciones, un estudio de mercado ayuda a comparar cómo se encuentra la empresa respecto a su competencia, qué aptitudes se pueden desarrollar desde el ámbito interno, los procesos y la gestión, para mejorar de esta manera el alcance, disminuir los factores críticos y de esta forma, aumentar la producción y por lo tanto la rentabilidad de una pyme (Ríos, 2011).

Por consiguiente, con el examen realizado por la auditoría, se determina qué tan eficiente ha sido la planeación dentro de una empresa, así como mantener un control, y una buena gestión de los recursos, la auditoría de gestión integra la auditoría interna y externa, esto 
implica un mayor alcance metodológico en varios ámbitos empresariales, la auditoría interna en conceptos generales se sintetiza como la auditoría de administración que es la encargada de factores tales como la estructura interna, los objetivos, planes, procedimientos y funciones de la empresa, su fin es tener un control, realizando una revisión dentro de este entorno. Con esta revisión se pueden llevar a cabo informes y análisis para determinar la situación de las operaciones, en términos generales un control de calidad. La implementación de estos controles puede significar una manera de aseguramiento en el cumplimiento de los objetivos y la implementación de los recursos adecuados que la empresa tiene destinados (Gras \& García, 2015).

En concordancia con la auditoría de gestión se encuentra la auditoría operativa, la cual está encargada del control y cumplimiento de objetivos propuestos respecto a decisiones tomadas por la empresa, con el fin de conocer desviaciones en las metas inicialmente propuestas que producen que planes trazados no sean cumplidos. La auditoría operativa produce información que es llevada a la gerencia y determina la eficiencia en la obtención y manejo de los recursos. El implementar un plan de auditoría de gestión requiere de diversas etapas de preparación, en el que los objetivos y técnicas a utilizar son el camino por el cual va a estar enfocado el plan de auditoría, pues debe estar ampliamente alineada con las metas para de esta manera garantizar el cumplimiento de estas (Gras \& García, 2015).

Según Hidalgo M. Proaño (2011) "En términos generales se entiende por PYME que es una empresa con ventas y volúmenes de producción moderados y que no cuentan con altos capitales y tampoco disponen de un número de personal elevado." Según lo dicho por el autor las PYMES son pequeñas y medianas empresas que no tienen una cifra de capital muy alta y que no cuentan con un número de trabajadores elevado.

Las pymes requieren de la auditoria de gestión ya que con esta se evalúan la eficacia y eficiencia con que se están manejando los recursos de la empresa, en caso que no se den los objetivos esperados se planteara nuevos planes o estrategias para lograr estos objetivos.

Según Millano (como se citó en Leal M, 2015) "afirma que la auditoría de gestión comprende un conjunto de métodos y medidas adoptadas dentro de una organización para verificar la exactitud y veracidad de su información administrativa, al promover la eficiencia, eficacia y efectividad en los procesos y operaciones". En otras palabras la auditoria de gestión es una agrupación de procedimientos que permite evaluar el grado de cumplimiento de la misión, visión, objetivos, entre otros, que permiten cuantificar el nivel de eficacia e impacto tiene en determinado sector el cual se está analizando, con objetivo de enfrentarse a cambios fluctuantes que podrían afectar severamente a la organización, con el objetivo de convertir sus procesos a procesos eficientes y constituirse en una organización competitiva teniendo un personal ampliamente capacitado para la resolución de cada uno de los problemas que se presenten, así como para el manejo de recursos y una eficacia en su administración.

Para Reyes A. (como se citó en López J, Rodríguez C, 2018) "Los objetivos de la auditoria de gestión son los siguientes: a) Establecer el estado inicial de la empresa b) Revisar los objetivos y planes de la organización c) Vigilar la existencia de políticas adecuadas y el cumplimiento de las mismas d) Comprobar la confiabilidad de la información y de los controles establecidos e) Verificar la existencia de métodos o procedimientos adecuados de operación y la eficiencia de los mismos 


\section{2}

f) Comprobar la utilización adecuada de los recursos".

Bravo M. \& Bravo S. (2018) afirman "Cuando se ejecuta una auditoría de gestión es necesario cumplir de forma sistemática y cíclica las 4 fases básicas de la mayoría de auditorías incluida la de gestión; basados en el popularizado ciclo de Deming, sin embargo fue creado por Walter Shewhart (1920), pero se hizo próspero gracias al populismo que logró en Japón por Deming, por lo que es más conocido como Ciclo de Deming, que como el de Shewart; el ciclo "Planificar- Hacer-Verificar-Actuar" (PHVA)." En base a lo mencionado anterior dicho por el autor determina que dicho ciclo de Deming tiene en cuenta los aspectos, en su primera etapa planea los detalles, esto hace referencia a la formulación de los objetivos, misión, visión entre otros, la segunda etapa es hacer, esta se basa en la planeación y consiste en desarrollar los planes u objetivos planeados y propuestos en la etapa anterior, en la tercera etapa se encarga de verificar, medir y analizar cómo se establecieron los procesos anteriormente planeados y como última etapa es la del actuar la cual corresponde a establecer medidas correctivas en caso de que existan controversias entre el nacer y lo planeado, con base a esto se establece un plan de mejoramiento con medidas correctivas para lograr los objetivos planteados en la empresa. Por otra parte la auditoria de gestión conlleva a la rentabilidad ya que si se tiene una buena gestión y se corrigen las fallas que se estén presentando en las empresas se podría tener una alta rentabilidad, pero si sucede lo contrario la rentabilidad bajaría.

Para Zamora (como se citó en Marmanillo C, Fransheska G, 2018) "Establece la rentabilidad, como una relación entre la utilidad y la inversión que se realiza para obtener la utilidad, si se baja la cifra de la inversión con los ingresos generados se emplea un monto del cual se puede concluir que este monto es la ganancia de la empresa durante ese periodo; la rentabilidad a su vez mide la eficiencia que ejerce la gerencia con respecto a las ventas realizadas en un periodo determinado, dando como resultado la utilidad". En otras palabras la rentabilidad es lo restante entre la utilidad y lo invertido en un producto o servicio de una empresa esta varía de acuerdo a la eficiencia de los trabajadores de una empresa pero por otro lado también influye los costos de dicho producto o servicio.

En la auditoria de gestión y la rentabilidad influyen muchos factores como los expuestos por Martínez (como se citó en Zelada A, Madelleine M, 2017) "Se determinó, que sus colaboradores no se comprometen con sus responsabilidades por lo que el resultado de sus servicios no son los esperados, y al no mantener un manual de procesos actualizado, es necesario efectuar una reingeniería a los procesos del área de Recursos Humanos, parte sensible de la compañía."

Con base a lo anterior mencionado por el autor se puede deducir que bajo el estudio que se realizó a esta compañía se concluye que en esta empresa los colaboradores o empleados no cumplen con sus actividades correspondientes por lo cual los procesos no son realizados con la mayor eficiencia, en este caso la auditoria de gestión tendrá como objetivo corregir y dar solución a esta problemática, con el fin de lograr los objetivos propuestos por la compañía y por ende lograr una alta rentabilidad (Bravo \& Bravo, 2018).

En la Auditoría de Gestión está integrada a la evaluación del uso eficiente de los recursos, tanto humanos como físicos, así como una valoración de varios métodos de operación, también recomendaciones para soluciones de problemas.

La Auditoría de Gestión es una valoración crítica que analiza las actividades que se 
llevan a cabo en el proceso de gestión de una organización, con el objetivo de establecer las medidas con las que se realiza, el esfuerzo, rapidez, profesionalismo, igualdad y excelencia se realiza el gasto de la empresa, la adaptación y acomodación de los métodos de indagación y vigilancia.

Los siguientes son los objetivos de la auditoría de gestión:

- Evaluar y determinar que las pymes acepten y desempeñen sus criterios de políticas tanto internas como externas.

- Verificar en las PYMES el manejo apropiado de los recursos materiales, económicos y el talento humano.

- Revisar que las pequeñas y medianas empresas elaboren con gran sentido de pertenencia

- Examinar que se cumplan con las metas trazadas.

- Verificar que la información Administrativa usada sea correcta.

Marques (citado en Matheus, 2015) manifiesta que: "La perspectiva tradicional de la auditoria apoyado en la realidad de controles, en las transacciones todo ello desde una perspectiva de contabilidad es un abordaje reduccionista, sin la posibilidad de abarcar un concepto de auditoria más unido con el futuro que con el pasado".

En la actualidad la auditoría se ha transformado en un instrumento importante para la gestión de cualquier empresa, es por esa razón que nace la obligación de evaluar a una organización en otras ramas diferentes a lo financiero, por lo cual se define a la auditoria como: el medio de lograr y valorar imparcialmente en un tiempo específico pruebas relativas a la siguiente temática: el registro financiero, la base del control interno financiero, el acatamiento de las leyes y ordenamientos pertinentes y la gestión ordenada para conseguir las metas propuestas con el ánimo de comunicar sobre el grado de reciprocidad entre estos temas y los juicios o señales establecidas para su revisión (Blanco, 2012).

Sánchez (2006) expresa que "la auditoria es un examen completo sobre la estructura, las transacciones y el desempeño de una entidad económica, para contribuir a la oportuna prevención de riesgos, la productividad en la utilización de los recursos y el sometimiento permanente de los mecanismos de control implantados por la administración."

En la auditoría actual, se comienza a lograr satisfacción de auditoría desde los niveles de Dirección de la empresa, donde se prueba el cumplimiento y la aplicación de controles por parte de los ejecutivos. El plan de trabajo se realiza en periodo real por parte del auditor en donde verifica cada una de las partes de la estructura hasta llegar a las bases individuales, permitiendo flexibilidad e importancia para los sistemas y controles (Ramírez, 2013).

La auditoría de gestión brindara apoyo a la compañía para lograr una adecuada y oportuna que permita mostrar las decadencias y aspectos a corregir en las fases para alcanzar unas ganancias y el aumento empresarial.

La auditoría de gestión permite conocer el nivel real de la administración y situación de la empresa; una herramienta, que a la vez ayudará a asesorar la gerencia y nos guiará en la adecuación necesaria para las metas que surjan en un contexto cambiante (Arriaga, 2014).

De otro modo una auditoria de gestión es el estudio minucioso de las actividades de una empresa o una parte de ella en contacto con metas específicas, tal verificación tiene 


\section{4}

3 propósitos principales: valorar el manejo y reconocer las oportunidades de mejorar y realizar pautas de mejoramientos y promover acciones. (Vásquez y Pinargote, 2018).

Para Arias González (2018) “La auditoría de gestión está basada en conseguir etapas que originen una alta fragilidad y economía fundada en una regulación, así como de mostrar fallas o distorsión que generen un impacto negativo que afectan al desarrollo normal de las funciones empresariales $u$ organizacionales". La Auditoría de Gestión tiene como propósito, comunicar a la gerencia si las operaciones se llevan a cabo o no de manera que cumplan con las políticas, estrategias y propósitos estables, orientados a la búsqueda de las metas de la Administración.

\section{Conclusiones}

En conclusión, el análisis de los conceptos y definiciones planteados por varios autores, ayudaron tener conocimientos claros y concisos sobre el tema propuesto en el anterior ensayo, y como conclusión se determina que la auditoria de gestión la cual busca evaluar el grado de eficiencia de las organizaciones, incide en la rentabilidad de las pymes ya que al una empresa tener una estructura organizada de sus trabajadores y el buen manejo de sus recursos disponibles, se lograra una cifra elevada en la rentabilidad de esta. las pymes deben realizar auditorías de gestión para determinar las deficiencias que se tengan en las organizaciones, y en base a esto buscar soluciones para corregir estas fallas que se vienen presentando, o por otro lado también se pueden realizar cambios en los sistemas y áreas de las empresas siempre y cuando se vea reflejado el mejoramiento de las organizaciones, en la auditoria de gestión son indispensables los factores estratégicos como lo son el uso de las tecnologías, mantener procesos contantes en la evolución de la empresa, realización de capacitaciones, mejoramiento de procesos administrativos mediante la evolución, mantener la generación de estrategias para el mejoramiento de factores críticos para lograr los objetivos planteados en una organización que también busca obtener una elevada cifra en la rentabilidad.

\section{Referencias}

Arias González, I.P. (2018) Auditoría un Enforque de Gestión

Arriaga Ramírez, J. (2014). La importancia de la auditoría de gestión

Blanco, Y. (2012). Auditoría integral, normas y procedimientos. Bogota: Ecoe Ediciones

Gras-Gil, E., Marín-Hernández S. y GarcíaPérez de Lema, D. (2015). Auditoría interna y deficiencias de la información financiera en el sector bancario español. Revista de Contabilidad, 18(2)

Fababa, L. (2020). Auditoría, tributación, finanzas y rentabilidad de las micro y pequeñas empresas nacionales del sector comercio, rubro farmacia del distrito de Manantay. Universidad Católica de los Ángeles Chimbote

Martínez López, G. (2011). Auditoría de Gestión y su Incidencia en la Rentabilidad de la Compañía Itaferi Corporation S.A. Universidad Técnica de Ambato. Ecuador

Hidalgo M, Proaño C, Sandoval M, (2011) "evaluación del uso de las tics en el desempeño de las pymes ubicadas en la zona urbana de la ciudad de latacunga" escuela politécnica del ejército extensión latacunga, Latacunga

Leal Romero, M. (2015) "Minirrevision de literatura sobre la auditoria de gestión como una herramienta eficiente y eficaz para las empresas", Pontifica Universidad Javeriana, 


\section{Bogotá Colombia}

Leal, R. (2015). Minirrevisión de literatura sobre la auditoría de gestión como una herramienta eficiente y eficaz para las empresas. Pontificia Universidad Javeriana, Facultad de Ciencias Económicas y Administrativas, Contaduría Pública

López-Cango, J. y Rodríguez-Mora, C.M. (2018) "Aplicación de auditoria de gestión y su efecto en la rentabilidad de empresa importadora Romhertex S.A.C”, Universidad señor de Sipan, Pimentel, Perú

Bravo Avalos, M.B., Bravo Avalos, S.P. \& López Salazar, J. L. (2018) "Importancia de la auditoría de gestión en las organizaciones.", Revista Observatorio de la Economía Latinoamericana. Disponible en: https://www.eumed.net/rev/ oel/2018/05/auditoria-gestion-organizaciones. ht m l//hd1.handle.net/20.500.11763/ oel1805auditoria-gestion-organizaciones

Marmanillo Chuco, F.G. (2018) "Auditoría financiera y su incidencia en la rentabilidad de las empresas comercializadoras de productos químicos en Lima Norte, Perú 2018”, Universidad Cesar Vallejo, Callao Perú

Matheus, I. (2015). Aproximación a un Cambio de Enfoque de la Auditoría Tradicional Hacia la Auditoría Holística: Una Mini Revisión de Literatura

Palomo González, M.A. (2005). Los procesos de gestión y la problemática de las PYMES. Ingenierías, VIII (28)

Ramírez, J. (2013). Herramientas para la Evaluación del Riesgo y su Relación con la Auditoría Integral. Adversia Revista virtual de estudiantes de contaduría pública, 1-14

Ríos Guato, L. (2011). Evaluación al Control Interno en el Proceso de Ventas y su Incidencia en la Rentabilidad de la Empresa Megamultisuelas en el Segundo Semestre del Año 2010. Trabajo de Graduación. Universidad Técnica de Ambato. Ecuador

Sánchez, G. (2006). Auditoria de estados financieros Segunda Edicion. Mexico: Pearson Educación

Soy-Aumatell, C. (2003). La auditoría de la información, componente clave de la gestión estratégica de la información. El profesional de la información, 12(4), 261-268

Vásquez Giler, M. y Pinargote Vásquez, N. (2018). Auditoría de gestión: una herramienta de mejora continua. Manta, Ecuador: Universidad Laica Eloy Alfaro de Manabí

Zelada Arqueros, M. M. (2016) "Auditoria de Gestión y su incidencia en la Rentabilidad de la Empresa Comercializadora y Servicios Bodserg S.A.C. Año 2016.”, Universidad Cesar Vallejo, Trujillo Perú 